\title{
IMPLEMENTAÇÃO DA PRODUÇÃO ENXUTA EM OPERAÇÕES HOSPITALARES: CASO DO INSTITUTO ONCOLÓGICO DOUTOR ARNALDO VIEIRA DE CARVALHO
}

\section{IMPLEMENTATION OF LEAN PRODUCTION IN HOSPITAL OPERATIONS: CASE OF THE ONCOLOGY INSTITUTE DOUTOR ARNALDO VIEIRA DE CARVALHO}

\author{
Tatyana Karla Oliveira Regis* E-mail: tatyana regis@yahoo.com.br \\ *Universidade Federal da Paraíba (UFPB), João Pessoa, PB
}

Resumo: Este artigo tem como objetivo analisar a implementação da produção enxuta (PE) no fluxo do paciente quimioterápico e radioterápico, assim como propor diretrizes para o desenvolvimento da cultura enxuta em uma operação hospitalar. A pesquisa classifica-se como qualitativa, aplicada e exploratória, e foi realizada por meio de um estudo de caso em um hospital do estado de São Paulo. A descrição do caso esclarece como ocorreu o processo de implementação da PE no hospital evidenciando o método e técnicas aplicadas. Fundamentando-se na literatura e no estudo de caso foi possível propor 12 diretrizes para implementação da produção enxuta em operações hospitalares, as quais, servem como subsídio às operações hospitalares que desejarem implementar a PE desenvolvendo melhorias em qualidade e produtividade.

Palavras-chave: Produção Enxuta. Operações de Serviços. Operações Hospitalares.

Abstract: This article aims to analyze the implementation of lean production in the flow of the chemotherapeutic and radiotherapeutic patient, as well as to propose guidelines for the development of lean culture in a hospital operation. The study was classified as qualitative, applied and exploratory, and was performed through a case study in a hospital in the state of São Paulo. The description of the case explains how the process of implementation of lean healthcare in the hospital occurred, evidencing the method and techniques applied. Based on the literature and the case study, it was possible to propose 12 guidelines for the implementation of lean production in hospital operations, which serve as a subsidy to hospital operations that wish to implement lean healthcare by developing improvements in quality and productivity.

Keywords: Lean Production. Service Operations. Hospital Operations.

\section{INTRODUÇÃO}

Embora a PE seja mais disseminada na manufatura, tem crescido a sua aplicação em operações de serviços e alguns pesquisadores já se dedicam a estudar a sua implementação nos serviços públicos, de varejo, saúde, call center, hotéis, aeroportos, etc. (YASIN; WAFA; SMALL, 2001; BARLOW, 2002; KOLLBERG; DAHLGAARD; BREHMER, 2007; PIERCY; RICH, 2009; PHENG; ARAIN; FANG, 2011). 
Dentre as operações de serviços que têm implementado a PE destaca-se o setor de saúde, no qual a PE é conhecida pela denominação Lean Healtchcare. Souza (2009) realizou uma revisão sistemática da literatura sobre PE nos serviços de saúde, na qual analisou mais de 90 publicações em mais de 10 países desde o ano de 2002, concluindo que a maioria das aplicações da PE em saúde ocorreu nos EUA (57\% das obras pesquisadas), seguido do Reino Unido (29\%) e começa a aparecer de forma mais consistente na Austrália (4\%) e no cenário internacional (outros países 9\%).

A implantação da PE nos serviços de saúde também tem sido popular na Inglaterra e tem ocorrido de diferentes formas que vão desde a exploração experimental na forma de aprender com os outros (hospitais e organizações de outros setores) até por meio de uma abordagem sistêmica alinhada à estratégia. Neste trabalho, abordagem sistêmica refere-se ao enfoque holístico, ou seja, abrange toda a organização. Em 2007, 80 hospitais ingleses (53\%) citaram a implementação da PE em seus relatórios de desempenho anuais e em 2009 esse número subiu para 111 (78\%) (BURGESS; RADNOR, 2013).

Em se tratando especificamente dos serviços de saúde no Brasil, Araújo (2005) afirma que o setor de saúde no país está marcado por custos crescentes na assistência juntamente com uma piora na qualidade dos serviços e restrições de acesso. O relatório do Desempenho Hospitalar Brasileiro afirma que no Brasil o setor de saúde gasta mal, desperdiça recursos e é mal gerenciado. (LA FORGIA; COUTTOLENC, 2009).

No Brasil algumas organizações do setor de saúde já iniciaram a implantação da PE, estando a maioria localizada no estado de São Paulo (BATAGLIA, 2013; SALVI, 2013). Dentre estas, destacam-se por seus resultados: o Instituto de Oncologia do Vale, reduzindo $50 \%$ de horas extras e aumentando a demanda em mais de 50\% com uma variação de pessoal de 5\%, a rede D'Or São Luiz, reduzindo o tempo de espera do paciente para atendimento no setor de emergência e o hospital São Camilo, que na unidade de Pompéia, obteve em 2012 uma redução de 200 mil dólares ao aplicar os princípios enxutos ao serviço de apoio ao diagnóstico e tratamento, além de uma redução de estoque de 8.557 para 2.283 filmes na área de imagens do hospital, representando uma economia de $R \$ 71$ mil (BATAGLIA, 2013; SALVI, 2013). 
Uma operação hospitalar reúne um conjunto de diferentes processos interligados, dentre os quais, destacam-se: serviços de enfermagem, nutrição e dietética; arquivamento médico e estatístico; assistência social; diagnóstico e tratamento; apoio ao diagnóstico e tratamento; e serviços de farmácia (SOUZA; MOZACHI, 2009; CAVALLINI; BISSON, 2010).

Ao analisar a literatura, verificou-se a evidência de aplicações da PE em operações de serviços de saúde, entretanto, as pesquisas focam os resultados em detrimento do caminho percorrido na implementação da cultura enxuta, ou seja, não deixam claro quais as melhores técnicas, métodos ou modelos de aplicação.

Dessa forma, diante do crescimento da aplicação da PE às operações de serviços e do desenvolvimento inicial da mentalidade enxuta nos serviços de saúde do Brasil, este artigo se propõe a responder a seguinte pergunta: Como implantar a produção enxuta no fluxo do paciente quimioterápico e radioterápico?

Para atender a este objetivo, foi estudada a literatura referente à produção enxuta em serviços, operações hospitalares e produção enxuta em operações hospitalares. Posteriormente, foi realizado um estudo empírico em um hospital filantrópico que presta assistência médica oncológica, situado no estado de São Paulo. Assim, foi possível analisar a implementação da PE no fluxo do paciente quimioterápico e radioterápico, assim como propor diretrizes para o desenvolvimento da cultura enxuta em uma operação hospitalar.

\section{DESENVOLVIMENTO}

\subsection{Lean Healthcare}

Os serviços de saúde possuem o paciente como cliente e componente do processo até que o tratamento seja concluído, sendo assim é necessário satisfazer o paciente por meio da disponibilidade dos serviços, dos prazos de entrega curtos e da qualidade ao mesmo tempo em que a organização se torna eficiente e competitiva (ARONSSON; ABRAHAMSSON; SPENS, 2011).

Conforme a Organização Mundial da Saúde, o hospital é o estabelecimento de saúde cuja função é prestar completa assistência médica à população, tanto preventiva como curativa, incluindo serviços de assistência domiciliar, assim como fornecer preparo e treinamento aos profissionais de saúde e dedicar-se às 
pesquisas biossociais (MALAGÓN-LONDOÑO; MORERA; LAVERDE, 2000; CAVALLINI; BISSON, 2010).

O sistema hospitalar oferece variados serviços e reúne um conjunto de diferentes processos interligados que consomem variados recursos. Os processos são classificados em:

- Processos administrativos: recursos humanos, processamento de dados, finanças, faturamento, serviços gerais, segurança e medicina do trabalho, suprimentos e técnicos, etc. (SOUZA; MOZACHI, 2009; CAVALLINI; BISSON, 2010);

- Processos técnicos: Serviços de enfermagem, nutrição e dietética, arquivamento médico e estatístico, assistência social, diagnóstico e tratamento, apoio ao diagnóstico e tratamento, serviços de farmácia e etc. (SOUZA; MOZACHI, 2009; CAVALLINI; BISSON, 2010).

A Figura 4, a seguir ilustra alguns fluxos hospitalares.

Figura 1 - Fluxos hospitalares

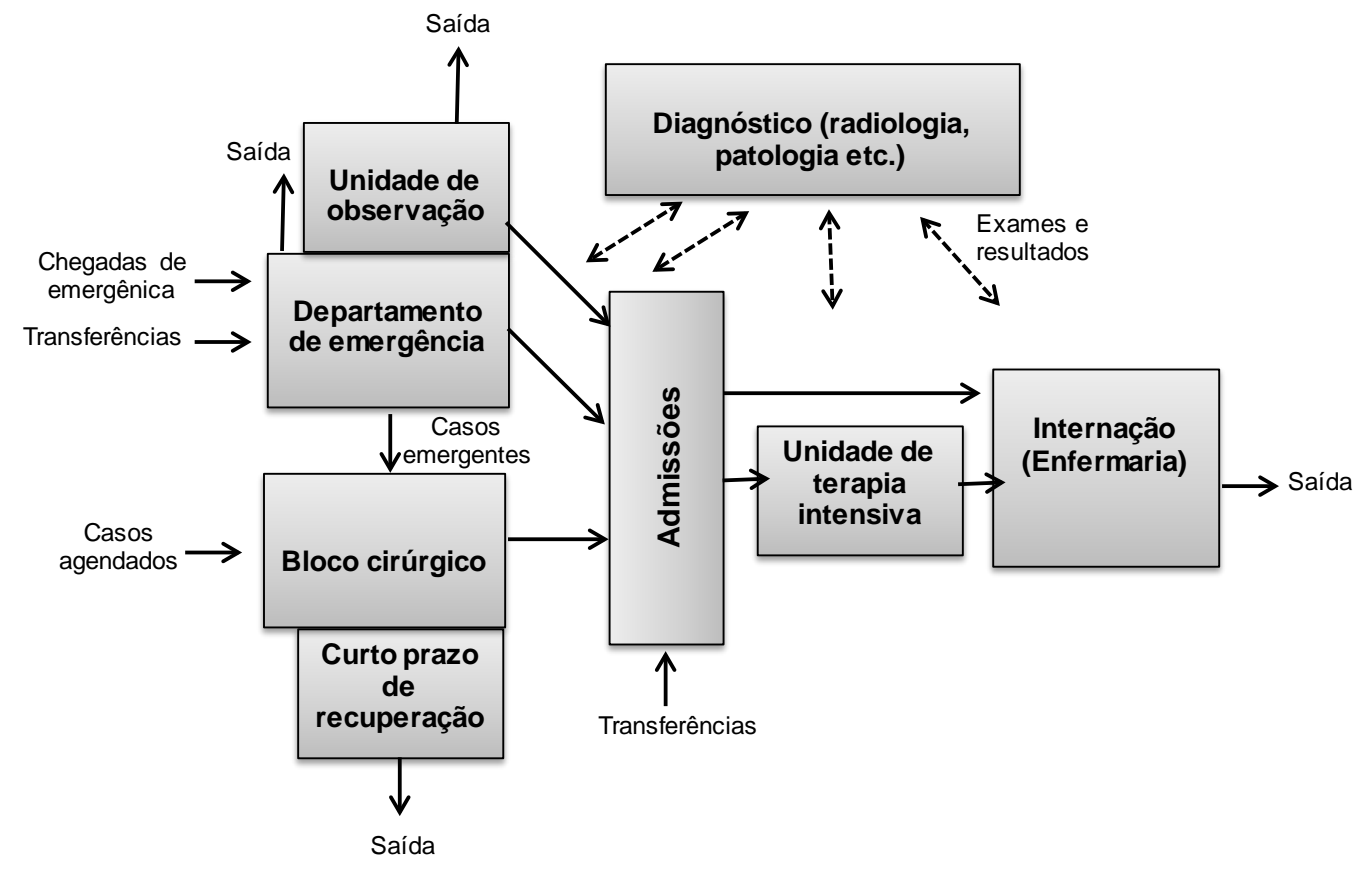

Fonte: Hopp e Levejoy (2013, p. 18).

*Linhas cheias representam fluxos de paciente e linhas tracejadas representam fluxos de informação.

Conforme Kollberg, Dahlgaard e Brehmer (2007) o processo principal do serviço é a razão de ser da empresa, é o que cria o valor desejado pelo cliente, no 
caso dos hospitais, os processos principais têm a função de promover ou recuperar a saúde do paciente como, por exemplo, os processos de diagnóstico e tratamento, enquanto que os serviços de alimentaçã e outros são apoiadores do serviço central.

O hospital, assim como qualquer empresa, deve desenvolver a qualidade de seus serviços por meio da gestão eficiente dos seus processos visando assegurar a sua competitividade (SOUZA; MOZACHI, 2009; LUONGO, 2011).

Neste sentido, a PE tem sido implementada nas operações hospitalares com o objetivo de promover a melhoria da qualidade e produtividade.

Shingo (1996) define a PE como um sistema de gestão da produção que visa à eliminação total das perdas. As perdas consistem em atividades que consomem recursos, gerando custo e não adicionam nenhum valor ao produto (SHINGO, 1996; ONHO, 1997; LIKER e MORGAN, 2006). Graban (2013) analisou as perdas combatidas pela PE, nos serviços de saúde, conforme são ilustradas no Quadro 1, a seguir.

A semelhança de Graban (2013), Jimmerson (2010) também descreveu as perdas da PE aplicadas aos serviços de saúde, entrentanto Jimmerson (2010) une o transporte e a movimentação em uma única perda, inclui a confusão presente nas organizações de saúde como uma perda que é capaz de ocasionar as demais.

A confusão reflete a falta de organização do processo e de conhecimento por parte dos trabalhadores. Ao reduzir a confusão aumenta-se a capacidade de trabalho, diminui-se a frustração dos trabalhadores e a ocorrência de erros (JIMMERSON, 2010).

O objetivo da PE é reunir um conjunto de princípios e técnicas visando à eliminação das perdas e a melhoria dos processos. Conforme Godinho Filho (2004), os princípios estão associados à filosofia da PE, enquanto que as técnicas são os meios pelos quais os princípios são atingidos. Abaixo estão relacionados os principais princípios e das técnicas da PE.

- Princípios: determinar o valor para o cliente, definir o fluxo de valor, manter o fluxo contínuo, produção puxada, integração da cadeia de fornecedores, foco na qualidade, gerenciamento visual, uso de tecnologia que atenda a funcionários e processos, desenvolvimento dos recursos humanos e melhoria contínua (SHINGO, 1996; GODINHO FILHO, 2004; WOMACK et al, 2005, LIKER, 2008). 
Quadro 1- Perdas nas operações hospitalares

\begin{tabular}{|c|c|c|}
\hline Perdas & Descrição & Exemplos hospitalares \\
\hline Falhas & $\begin{array}{l}\text { Atividades que não são realizadas } \\
\text { corretamente. }\end{array}$ & $\begin{array}{l}\text { Medicamento errado, prontuário } \\
\text { preenchido incorretamente, } \\
\text { carrinho de limpeza faltando um } \\
\text { item. }\end{array}$ \\
\hline Superprodução & $\begin{array}{l}\text { Fazer mais que o demandado pelo } \\
\text { cliente, ou produzir antes de surgir } \\
\text { a demanda. }\end{array}$ & $\begin{array}{l}\text { Realização de exames e de } \\
\text { procedimentos administrativos } \\
\text { desnecessários, entrega de } \\
\text { medicamentos aos pacientes antes } \\
\text { da hora. }\end{array}$ \\
\hline Transporte & $\begin{array}{l}\text { Movimentação excessiva de um } \\
\text { produto (paciente e materiais) ao } \\
\text { longo do sistema. }\end{array}$ & $\begin{array}{l}\text { Layout inadequado acarretando em } \\
\text { longos transportes de pacientes. }\end{array}$ \\
\hline $\begin{array}{c}\text { Excesso de } \\
\text { processamento }\end{array}$ & $\begin{array}{l}\text { Produzir com o nível de qualidade } \\
\text { superior ao demandado ou realizar } \\
\text { uma atividade desnecessária. }\end{array}$ & $\begin{array}{l}\text { Informações coletadas em } \\
\text { formulários, mas nunca utilizadas. }\end{array}$ \\
\hline Estoque & $\begin{array}{l}\text { Excesso de estoque e capital } \\
\text { imobilizado. }\end{array}$ & $\begin{array}{l}\text { Medicamentos fora da validade e } \\
\text { suprimentos vencidos. }\end{array}$ \\
\hline Movimentação & $\begin{array}{l}\text { Movimentos desnecessários dos } \\
\text { funcionários no sistema. }\end{array}$ & $\begin{array}{l}\text { Layout inadequado acarretando em } \\
\text { longos transportes dos } \\
\text { enfermeiros. }\end{array}$ \\
\hline Espera & $\begin{array}{l}\text { Tempo no qual não é realizado } \\
\text { nada de produtivo. }\end{array}$ & $\begin{array}{l}\text { Pacientes esperando por consultas } \\
\text { ou procedimentos como } \\
\text { radioterapia e quimioterapia. } \\
\text { Funcionários esperando devido a } \\
\text { desequilíbrios nas suas cargas de } \\
\text { trabalho. }\end{array}$ \\
\hline Talento & $\begin{array}{l}\text { Ocorre quando as competências } \\
\text { dos trabalhadores não são } \\
\text { utilizadas para melhorar o } \\
\text { processo. }\end{array}$ & $\begin{array}{l}\text { Funcionários desmotivados, não } \\
\text { apresentam sugestões de } \\
\text { melhoria. }\end{array}$ \\
\hline
\end{tabular}

Fonte: Graban (2013, p.45)

- Técnicas: mapeamento do fluxo de valor (MFV), just in time, kanban, autonomação (jidoka), 5s, padronização, nivelação da carga de trabalho (heijunka), tecnologia de grupo e layout celular, trabalhar de acordo com o takt time, troca rápida de ferramentas (TRF), controle de qualidade zero defeitos, manutenção produtiva total, controle visual, trabalho em equipe e trabalhadores multifuncionais, empowerment (autonomia) e kaizen (GODINHO FILHO, 2004; WOMACK et al, 2005; LIKER, 2008).

Bertani (2012) observa nove pontos importantes na implementação da PE em operações hospitalares. 
1. Definição do cliente: No contexto hospitalar definir o cliente não é uma tarefa simples, pois o hospital possui vários processos, mantenedores, profissionais e pacientes, cada um destes podendo ser considerados clientes, entretanto o valor do serviço deve ser definido pelo cliente principal, o paciente.

2. Estrutura de implementação: Muitas vezes são formadas equipes ad hoc para realização de kaizen e implementação de melhorias pontuais. Em alguns casos é estabelecida uma equipe interna fixa para gerenciar o processo de implementação da PE, podendo ter um membro da alta gerência na equipe.

3. Estabelecimento de metas e objetivos: A maioria dos hospitais não definem metas e objetivos claros na fase inicial de implementação da PE.

4. Envolvimento de pessoas: Para a aplicação eficiente das técnicas enxutas é importante o envolvimento dos funcionários que atuam na operação que está sendo melhorada, podendo haver participação de funcionários de outros processos, áreas e setores. A participação de representantes da alta gerência também é importante.

5. Treinamento: É imprescindível o treinamento dos profissionais envolvidos nos processos de melhoria enxuta por profissionais já experientes.

6. Mapeamento da situação atual e desenvolvimento da situação futura: A maioria das aplicações da PE utilizam o mapeamento de fluxo do processo para identificação de perdas e implementação de melhorias.

7. Implementação de melhorias: Em geral as implementações ocorrem por meio da técnica kaizen.

8. Sustentabilidade das melhorias: A padronização é utilizada com a finalidade de sustentar as melhorias alcançadas, entretanto alguns hospitais definem um proprietário para cada fluxo que tem a responsabilidade de manter e revisar o trabalho. 
9. Melhoria contínua: A melhoria contínua se dá em ciclos, quando uma situação futura é implementada, ela se torna a situação atual, devendo posteriormente ser analisada e melhorada.

Em especial, em se tratando de lean healthcare, as datas das primeiras aplicações das técnicas enxutas são incertas, entretanto alguns pesquisadores citam o Virginia Mason Medical Center e o ThedaCare nos EUA, o Flinders Medical Centre, na Austrália e o Royal Bolton NHS Foundation Trust no Reino Unido como exemplos bem sucedidos da implementação da PE em serviços de saúde, os quais iniciaram a implantação da PE entre os anos de 2002 e 2003 (WOMACK et al., 2005; PAPADOPOULOS; RADNOR; MERALI, 2011; YEH et al., 2011; RADNOR; HOLWEG; WARING, 2012; CLARK, SILVESTER; KNOWLES, 2013).

Quanto ao surgimento dos primeiros trabalhos acadêmicos sobre a aplicação da PE aos serviços de saúde, Souza (2009) afirma que se especula que foram publicados pela NHS Modernisation Agency (Agência de Modernização do Serviço Nacional de Saúde) no Reino Unido nos anos de 2001 e 2002.

Souza (2009) ressalta também as publicações de Allway e Corbett (2002), que enfatizam a aplicação da PE aos serviços de uma forma geral; Bushell e Shelest (2002), que descreveram a aplicação das técnicas enxutas a um hospital de médio porte nos Estados Unidos; Feinstein, Grunden e Harrison (2002), que relataram que a Iniciativa Regional de Saúde de Pittsburgh implementou técnicas da PE nos processos de obstetrícia, cirurgia ortopédica, depressão e diabetes, com o objetivo de reduzir as infecções e os erros de medicação para zero. Entretanto nenhum destes trabalhos evidenciaram nitidamente a implementação da PE.

Analisando a literatura verifica-se um amplo acervo de pesquisas e aplicações das técnicas enxutas em diversos processos hospitalares.

Dickson et al. (2009) investigam a implementação da PE nos processos de emergência, por meio de quatro hospitais com processos e culturas diferentes. De acordo com Dickson et al. (2009), os processos de emergência utilizaram o evento kaizen para iniciar a implementação da PE em três etapas: documentação do processo por meio do mapeamento do fluxo de valor; análise do mapeamento do fluxo atual com a finalidade de descobrir as perdas e de elaborar o MFV do estado futuro; e, teste de ideias para atingir o estado futuro idealizado. 
Segundo Dickson et al. (2009) após a implementação inicial, três dos quatro departamentos de emergência demonstraram melhorias no processo e na satisfação do paciente apesar do aumento da demanda.

Carter et al. (2012) estudaram a aplicação da técnica MFV às operações clínicas do processo de internação hospitalar do Komfo Anokye Teaching Hospital (KATH) em Gana. O KATH está localizado na segunda maior cidade de Gana e tem mais de 800 leitos hospitalares, atendendo a dois terços do norte de Gana (CARTER et al., 2012).

O programa de melhoria iniciou com um evento kaizen com duração de três semanas, no qual foi formada uma equipe multidisciplinar para elaborar o mapeamento do fluxo de valor, analisar a causa raiz das perdas, planejar o mapa do estado futuro e implementar as melhorias (CARTER et al., 2012).

Os processos cirúrgicos também foram foco da implementação da PE. Nesse sentido, Teichgräber e Bucourt (2012) investigaram sob a ótica dos princípios enxutos, o processo de aquisição e implantação de stents endovasculares nos serviços de cardiologia intervencionista. Após análise do MFV atual, ou pesquisadores afirmam que das trezes atividades do processo, apenas duas adicionavam valor ao serviço (suprimento de stents e implante de stents). Foi elaborado o MFV futuro, eliminando as perdas do processo por meio do princípio da produção puxada (TEICHGRÄBER; BUCOURT, 2012).

LaGanga (2011) examinou por meio de pesquisa-ação, as melhorias que foram obtidas com o primeiro projeto enxuto realizado em uma organização ambulatorial de saúde mental. Inicialmente foram aplicadas as técnicas kaizen e mapeamento do fluxo de valor, após um ano de implementação da técnica mapeamento do fluxo de valor, obtiveram-se os seguintes resultados: aumento de $11,52 \%$ do número de consultadas agendadas, aumento de $26,6 \%$ do número de consultas mantidas (LAGANGA, 2011).

Radnor, Holweg e Waring (2012) investigaram a aplicação da PE, por meio de estudo de caso realizado em quatro hospitais ingleses, concluindo que a PE concentrou-se na utilização de técnicas específicas que proporcionaram resultados rápidos ao invés de focar a sua abordagem sistêmica, vinculada ao planejamento estratégico, assim como o controle e melhoria da prestação de serviços no nível interorganizacional. 
Sullivan et al. (2014) analisaram a implementação da PE na farmácia de um hospital de oncologia nos EUA. Por meio do MFV foram eliminadas seis atividades que não agregavam valor ao serviço, assim, o lead time do fluxo passou de 91 minutos para 71 minutos. A programação do ambulatório e da farmácia foram melhoradas, o novo fluxo de trabalho foi padronizado e os funcionários treinados (SULLIVAN et al, 2014).

Papadopoulos, Radnor e Merali (2011) por meio da teoria ator-rede, exploraram a dinâmica na implementação da PE em um hospital do Reino Unido. Os pesquisadores investigaram a aplicação da PE na unidade de patologia (laboratórios), a qual tinha como objetivos: redução das perdas; do tempo de resposta aos pacientes e dos custos; e, melhoria da qualidade e da moral dos funcionários (PAPADOPOULOS; RADNOR; MERALI, 2011).

Segundo Radnor, Holweg e Waring (2012) a competição, a política contraditória, as regulamentações, a presença de grupos profissionanis poderosos com conhecimentos especializados e formas de trabalho definidas aumentam a complexidade dos processos hospitalares e dificultam a implementação sistêmica da PE na organização e na cadeia de suprimentos.

Burgess e Radnor (2013) avaliaram a implementação da PE nas operações hospitalares por meio da análise dos relatórios de desempenho anual dos hospitais ingleses e constataram que a extensão da implementação da PE vai desde a utilização de ferramentas como o MFV e do programa 5S a um planejamento da adoção dos princípios enxutos vinculados a estratégia.

Soliman e Saurin (2017) analisaram as barreiras e dificuldades em lean healthcare por meio de entrevista com três acadêmicos e dois profissionais, assim como por meio de observações e coleta de dados em dois hospitais. Os autores concluíram que as barreiras e dificuldades são dinâmicas e que surgiram novas barreiras importantes, e que devem ser considerados: dificuldade de experimentação, desequilíbrio na entrega de valor; e abordagem inadequada.

A maior parte das evidências mostraram que a PE foi aplicada por meio de um pequeno projeto de melhoria enquanto que apenas cinco hospitais alegaram implementar a PE como parte do planejamento estratégico do hospital, alinhada com os objetivos operacionais e com a mudança de cultura, na direção de uma uma filosifia de trabalho focada na melhoria contínua dos processos (BURGESS; 
RADNOR, 2013). Assim, o resultado da pesquisa de Burgess e Radnor (2013) reforçam as conclusões de Radnor, Holweg e Waring (2012).

\subsection{Metodologia}

Esta pesquisa é classificada como qualitativa e exploratória, uma vez que foi aplicado um estudo de caso e o resultado foi uma maior compreensão a respeito da aplicação da PE às operações hospitalares, que envolveu levantamento bibliográfico e entrevistas com pessoas que tiveram experiências práticas com o problema pesquisado. (SILVA; MENEZES, 2005).

O estudo de caso é aplicado quando se deseja obter uma compreensão mais profunda sobre o objeto pesquisado (YIN, 2005).

A primeira fase da pesquisa consistiu no desenvolvimento teórico-conceitual, nesta etapa a pesquisadora buscou a compreensão dos temas: PE, operações de serviços e PE em serviços de saúde.

Fundamentando-se na literatura foi possível estabelecer as seguintes categorias de análise: fatores motivacionais, estrutura de implementação, história e trajetória de implementação, capacitação dos recursos humanos, operações/processos, perdas nos processos, técnicas enxutas implementadas em cada um dos processos, resultados alcançados, comprometimento dos recursos humanos (alta gestão e operacional) e dificuldades. Estas categorias de análise auxiliaram na elaboração do instrumento de pesquisa.

Para o desenvolvimento do estudo empírico, foi selecionado o Instituto do Câncer Dr. Arnaldo Vieira de Carvalho (ICAVC) no estado de São Paulo. No estudo de caso foram utilizados os seguintes instrumentos de coleta de dados: entrevistas semiestruturadas, documentos que foram disponibilizados pelos hospitais e observações assistemáticas.

No ICAVC foram realizadas três entrevistas com líderes na implementação da PE. A primeira entrevista aconteceu com a coordenadora de qualidade, no dia 22/08/2014 e teve duração de 1 hora e 30 minutos. Entretanto, sentiu-se a necessidade de outra entrevista com a coordenadora de qualidade, com a finalidade de esclarecer algumas questões. Assim, no dia 03/09/2014 foi realizada a segunda entrevista, a qual teve duração de 1 hora. Após a entrevista, a pesquisadora pôde 
observar o processo de quimioterapia in loco, embora não dispusesse de um roteiro de observação pré-definido. Foram observados quadros de gestão visual, contendo o plano de trabalho, indicadores de desempenho, instruções de trabalho e controles de processos. A terceira entrevista foi realizada com a coordenadora do processo de radioterapia, no dia 12/09/2014 e teve duração de 1 hora e 5 minutos. As entrevistas foram direcionadas por um roteiro, gravadas e posteriormente transcritas.

Assim, foi analisada a forma como o hospital implementou a PE, considerando as categorias de análise apresentadas anteriormente e aplicando a análise de conteúdo.

\subsection{Resultados}

As próximas seções apresentam os resultados obtidos por meio do estudo de caso realizado no hospital Instituto do Câncer Dr. Arnaldo Vieira de Carvalho.

\subsubsection{Caracterização do hospital}

O ICAVC é uma instituição filantrópica fundada em 1920 na cidade de São Paulo-SP com o objetivo de prestar completa assistência médica oncológica à população.

A estrutura física do ICAVC é composta por dois prédios, intitulados como ambulatório e hospital. O ambulatório possui nove andares, cinco destes são destinados às atividades de consultas médicas e quatro às funções administrativas. O hospital concentra os tratamentos do câncer, tais como cirurgias, quimioterapias, radioterapias e exames, os quais são definidos por meio do atendimento ambulatorial.

O ICAVC é considerado de médio porte e atende o público adulto do estado de São Paulo e do Brasil por meio do Sistema Único de Saúde (SUS), contando com uma equipe de 600 funcionários entre assistenciais e administrativos. O ambulatório possui capacidade de 210 consultas por dia, funcionando das 7 às 17h, o hospital possui ala de internação com 76 leitos e 4 salas cirúrgicas. 


\subsubsection{A implementação da PE no ICAVC}

O ICAVC iniciou a implementação da PE em fevereiro de 2011. A motivação para a implantação da PE surgiu a partir do momento em que a consultoria Hominiss entrou em contato com o diretor administrativo do hospital e apresentou uma proposta para implementação explicando os benefícios potenciais desta.

A consultoria Hominiss ofereceu consultoria gratuita por um ano em busca da experiência de trabalho com a implantação da PE em operações hospitalares e da possibilidade de desenvolvimento da dissertação de um dos consultores. Conforme entrevista conduzida com a coordenadora de qualidade, o hospital aceitou a proposta, uma vez que necessitava da melhoria dos seus serviços e do aumento da rentabilidade. Após um ano, o hospital contratou a consultoria e esta permaneceu em atuação até fevereiro de 2014. Atualmente a mentalidade enxuta está sendo desenvolvida pelos funcionários do hospital sob a liderança da gestão da qualidade.

Dessa forma, de acordo com a coordenadora de qualidade o processo de implantação da PE foi vinculado ao planejamento estratégico, pois no momento inicial da implantação havia uma comissão de funcionários elaborando o planejamento estratégico e esta constatou que as metas da PE eram compatíveis com os objetivos estratégicos. Assim, foi elaborado o Balanced Scorecard (BSC) com objetivos da PE, para os processos de SAME (Serviços Ambulatoriais e Médicos Especializados), quimioterapia, radioterapia e exames. As figuras 2 e 3 a seguir apresentam o BSC dos processos de quimioterapia e radioterapia.

Segundo a coordenadora de qualidade, o ICAVC implementou a PE por meio da metodologia DMAIC, sendo o processo de implementação conduzido pelo diretor administrativo, pela coordenadora da qualidade e pela consultoria Hominiss. A metodologia DMAIC (Define, Measure, Analyze, Improve, Control) é utilizada para conduzir projetos Seis Sigmas, entretanto pode ser utilizada para gerir projetos de melhoria de uma forma geral (BERTANI, 2012).

A implantação da PE iniciou pelo fluxo do paciente quimioterápico, devido à quimioterapia ser um serviço chave no tratamento do câncer e também na rentabilidade do hospital. As metas foram: reduzir o tempo de espera, aumentar a capacidade do processo, e estruturar o setor de quimioterapia. Posteriormente, a PE foi implementada nos processos de radioterapia. 
Figura 2 - BSC-Quimioterapia

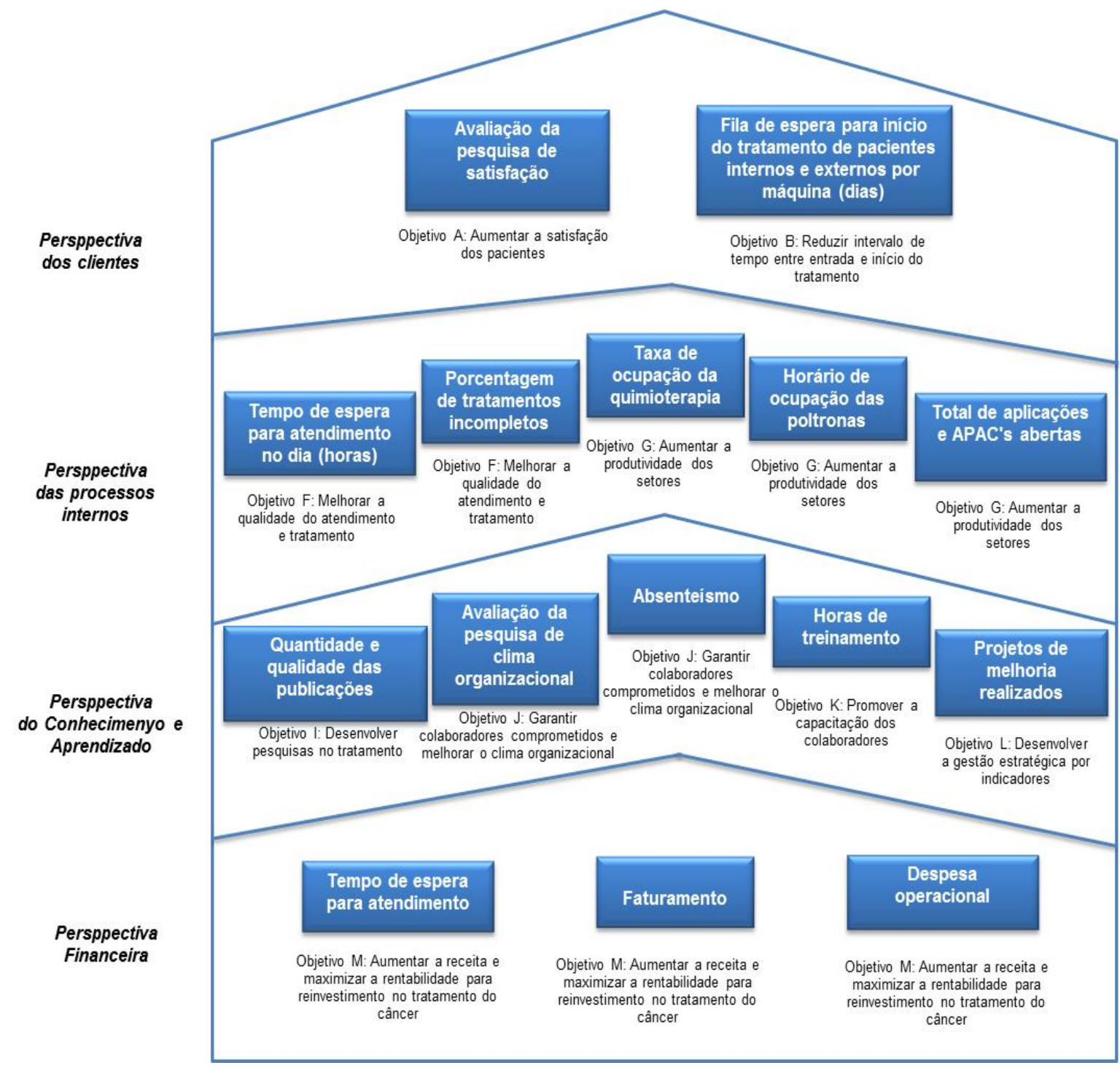

Fonte: ICAVC (2014)

A consultoria e coordenadora da qualidade realizaram reuniões de conscientização e treinamentos com os funcionários envolvidos no fluxo do paciente quimioterápico, por um período de três meses. Inicialmente foram treinados o gestor e o enfermeiro-chefe do setor de quimioterapia. Posteriormente foram treinados os demais funcionários envolvidos no processo em questão.

Os treinamentos dos funcionários eram realizados em um dia inteiro ou em uma semana, conforme os turnos de trabalho. Os treinamentos possuíam um caráter teórico e prático, a prática, portanto, ocorria por meio dos projetos de melhoria. Os treinamentos não tinham periodicidade definida, entretanto, eram realizados à 
medida que eram necessários ajustes nas melhorias realizadas ou novos projetos de melhoria.

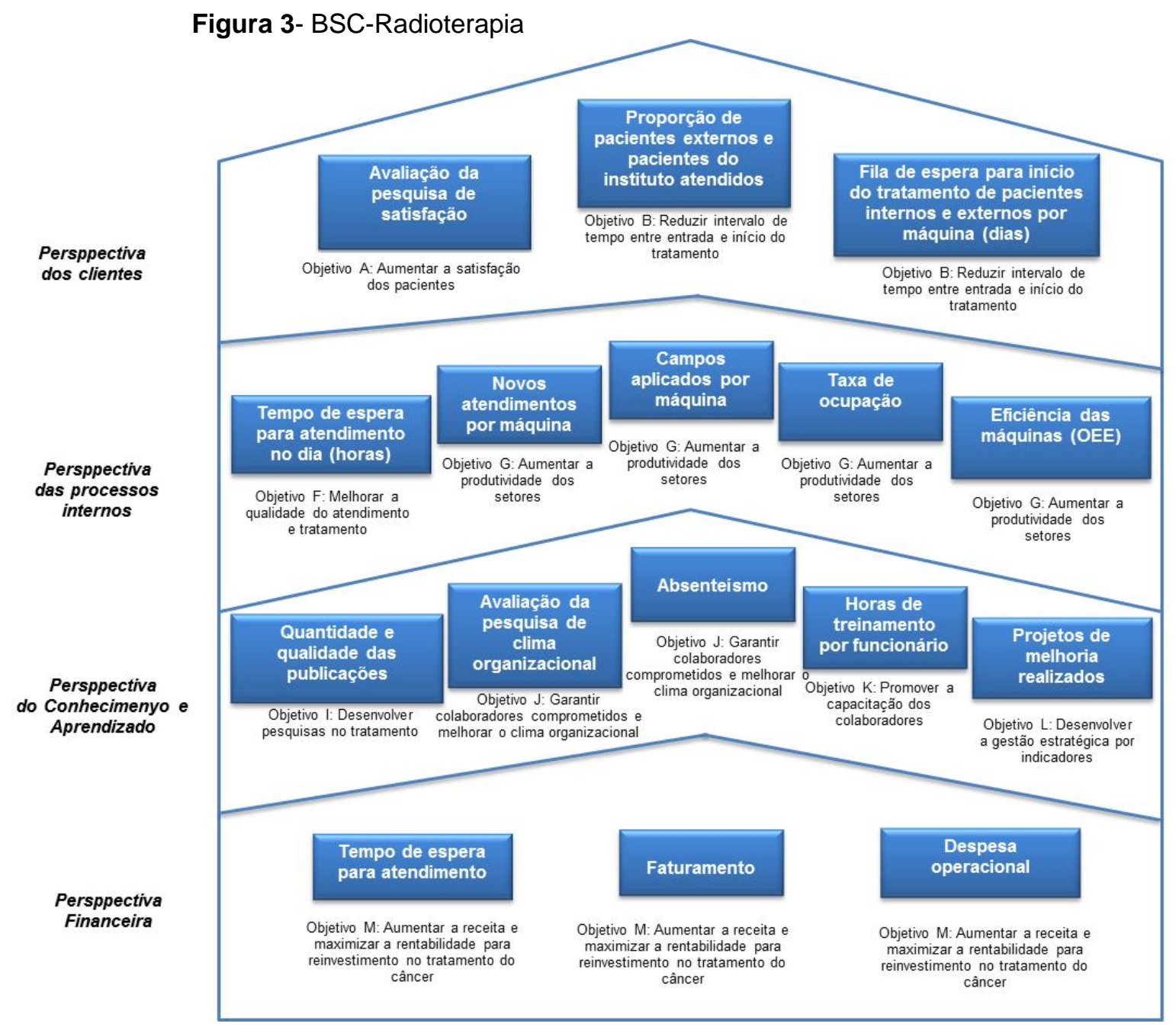

Fonte: ICAVC (20014)

As primeiras técnicas enxutas implementadas em todos os processos foram o MFV e o kaizen, uma vez que o MFV é uma técnica de diagnóstico da situação e que o kaizen é uma técnica que permite a formação de equipes de melhorias incrementais, por meio das quais as demais técnicas enxutas são implementadas.

Cabe ressaltar as perdas identificadas por meio do MFV realizado nos processos de quimioterapia e radioterapia, assim como as técnicas enxutas aplicadas com a finalidade de eliminar as perdas. Para tanto serão detalhados a seguir os processos de quimioterapia e radioterapia.

\section{- Quimioterapia}

Existe uma família de pacientes do fluxo do paciente quimioterápico, entretanto, de acordo com a coordenadora de qualidade, o ICAVC escolheu a família 
"novos pacientes sem exames" para iniciar a aplicação das técnicas enxutas. O fluxo dos novos pacientes quimioterápicos sem exames é o seguinte: triagem (o médico analisa se o paciente tem o câncer e define o tipo de tratamento: quimioterapia, radioterapia ou cirurgia), primeira consulta (médico analisa os exames do paciente e caso necessário, solicita novos exames), tomografia, ultrassonografia, exames de sangue, consulta (médico define o medicamento, dosagem e número de sessões), APAC (Autorização de Procedimentos de Alta Complexidade, fornecida pela secretária da saúde, e tem duração média de três meses) e quimioterapia.

O processo de quimioterapia operava das $7 \mathrm{~h}$ às $19 \mathrm{~h}$ de segunda a sexta-feira, e das $7 \mathrm{~h}$ às $13 \mathrm{~h}$ aos sábados, atendendo os pacientes por ordem de chegada. $\mathrm{O}$ agendamento das quimioterapias não considerava a capacidade do setor e o tempo de infusão dos medicamentos, assim, os pacientes eram orientados a não comparecer nos horários próximos ao fim do turno. Esta condição aliada ao fato do atendimento ser por ordem de chegada ocasionava maior demanda de pacientes e excesso de carga de trabalho pela manhã e alto tempo de espera dos pacientes.

A quimioterapia tem duração de $1 \mathrm{~h}$ a $8 \mathrm{~h}$, os pacientes cuja infusão durava $8 \mathrm{~h}$ muitas vezes ficavam por último, o que aumentava o tempo de espera destes pacientes. O processo de quimioterapia conta com duas salas, a primeira com 16 poltronas e a segunda com 9 poltronas, totalizando 25 poltronas. As falhas na programação das quimioterapias e na organização do trabalho da equipe da farmácia acarretavam no elevado tempo de carregamento das poltronas no início do turno. Muitos pacientes chegavam às $7 \mathrm{~h}$ no setor de quimioterapia, entretanto, apenas às $8 \mathrm{~h}$ e 30min as primeiras 16 poltronas estavam carregadas.

No processo de quimioterapia ocorria ociosidade das enfermeiras no início do turno, ocasionada pelo longo tempo de carregamento das poltronas, as enfermeiras ficavam esperando os pacientes para checagem dos sinais vitais e infusão medicamentosa.

Devido à falta de padronização do processo ocorria extravio de prescrições médicas, causando espera do paciente e perda de tempo dos funcionários. A ausência da padronização das atividades também acarretava na não inclusão de algumas quimioterapias no faturamento.

O ICAVC implementou técnicas enxutas com o objetivo de identificar as perdas no fluxo dos novos pacientes quimioterápicos sem exames e de eliminá-las. 
A implementação da PE iniciou com a elaboração de um projeto de melhoria, contando com a participação da equipe lean, composta por dois consultores da Hominiss e pela coordenadora de qualidade, assim como com a participação da enfermeira-chefe, das técnicas de enfermagem da quimioterapia, da farmacêutica responsável e de funcionários administrativos. O projeto de melhoria tinha como metas: melhorar a assistência reduzindo o lead time dos novos pacientes desde a triagem até o início do tratamento, reduzindo o tempo de espera do paciente, aumentando a capacidade do processo e estruturando o setor, assim como melhorando a situação financeira do hospital.

O projeto de melhoria iniciou com a aplicação das técnicas MFV e kaizen aos processos de triagem, consultas ambulatoriais, APAC e quimioterapia, procurando abranger todos os processos que reestabelecem a saúde do paciente. Após a elaboração do estado atual do processo e identificação das perdas, foi elaborado o estado futuro, o qual foi implementado por meio das técnicas: $5 \mathrm{~S}$, nivelamento da carga de trabalho, padronização, trabalhadores multifuncionais e controle visual, estas foram implementadas simultaneamente. Por fim foi implementada a técnica enxuta, controle de qualidade zero defeitos. As perdas do fluxo dos novos pacientes quimioterápicos sem exames são ilustradas no Quadro 2.

Após a aplicação das técnicas enxutas, o ICAVC começou a programar as quimioterapias considerando a capacidade do processo e o tempo de infusão dos medicamentos, alocando os pacientes que necessitam de maior tempo de quimioterapia nos primeiros horários. Os pacientes passaram a ter horário marcado, necessitando chegar com uma hora de antecedência para se identificar na recepção e aguardar a quimioterapia. O tempo de espera do paciente passou a ser de $1 \mathrm{~h}$, sendo este necessário devido à preparação dos medicamentos.

Foi definido que os mecânicos chegassem às $5 \mathrm{~h}$ e acionassem a capela, na qual os medicamentos são manipulados, assim como foram desenvolvidos planos de trabalho padrão para a equipe de farmacêuticos, estes devem iniciar a manipulação de medicamentos às $6 \mathrm{~h}$ e $30 \mathrm{~min}$. Estas ações foram tomadas com a finalidade de reduzir o tempo de carregamento das poltronas, e atualmente, às $7 \mathrm{~h}$ e 30 min. as primeiras 16 poltronas estão carregadas.

Também foram desenvolvidos planos de trabalho padrão para as atividades das técnicas e auxiliares de enfermagem e definido o rodízio de atividades. A cada 
semana ocorre o rodízio de atividades variando entre as atividades de verificação de sinais vitais e de acompanhamento da infusão de medicamentos.

\section{- Radioterapia}

O processo de radioterapia opera de segunda a sexta-feira, das $6 \mathrm{~h}$ às $23 \mathrm{~h}$ e 30 min., por meio de radioterapias convencionais (radiação na região) e conformacionais (3D, radiação localizada), com o tempo da radiação variando entre 15 e 20 minutos.

De acordo com a coordenadora da radioterapia o ICAVC dispõe dos seguintes equipamentos para realização da radioterapia: um acelerador 600/C (3D), o qual trata próstata e sistema nervoso central, atendendo a 110 pacientes/dia; um acelerador 6/100, o qual trata todas as especialidades e tem capacidade de 110 pacientes/dia, um cobalto para tratamento da mana, cabeça e pescoço.

A implementação da PE no processo de radioterapia contou com a participação da equipe lean, dos médicos, dos físicos, das técnicas de radioterapia, da coordenadora e do administrador do setor de radioterapia. Foi elaborado o projeto de melhoria do processo de radioterapia por meio da técnica kaizen, o qual tinha como meta, reduzir o tempo em que os pacientes esperam na fila até a aplicação da radioterapia.

Embora a meta do projeto inicial fosse uma melhoria pontual, foram realizados dois MFV's. O primeiro MFV avaliou o fluxo que inicia com a consulta oncológica e termina com a primeira aplicação da radioterapia, este possui a seguinte sequência: consulta oncológica, avaliação do radioterapeuta, planejamento da radioterapia, agendamento e aplicação da radioterapia. O segundo MFV analisou o fluxo que inicia com a chegada do paciente ao hospital até a aplicação da radioterapia.

Antes da implementação das técnicas enxutas, o segundo fluxo operava da seguinte maneira: o paciente ao chegar ao hospital se direcionava até uma técnica de radioterapia com seu cartão de identificação, a técnica procurava a ficha dele, conferia o agendamento, assinaturas e outras informações necessárias, entregava ao paciente a ficha com o respectivo número de ordem para aplicação da radioterapia. O paciente então aguardava a sua vez.

Assim, as técnicas tinham que desempenhar atividades burocráticas e administrativas, como também acompanhar a aplicação da radioterapia. Isto 
acarretava em maior tempo de ciclo da aplicação e maior tempo de espera do paciente em fila. Exemplo: enquanto a técnica estava verificando informações do paciente $A$, o paciente $B$ estava na sala de radioterapia esperando a técnica para aplicação e os demais pacientes aguardando na fila. Além das atividades administrativas das técnicas, a troca de roupas dos pacientes também contribuía para o maior tempo do paciente na fila.

Após a implementação da PE foi criada uma recepção para atender os pacientes, realizando as atividades administrativas, assim, as técnicas ficaram apenas com a atividade de assistir à aplicação da radioterapia. Foram criados vestiários dentro da sala do cobalto. Enquanto um paciente está saindo da máquina, outro já está se trocando para entrar na máquina. As implantações das melhorias enxutas estão em andamento, portanto ainda serão criados vestiários nas salas dos outros equipamentos do setor. Por meio destas mudanças no processo obteve-se uma redução do tempo de espera do paciente na fila de 50 min. para 20 min.

De acordo com o relato da coordenadora da radioterapia foram implementadas as seguintes técnicas enxutas: MFV, kaizen, padronização, gestão visual, 5S, TRF e CEP (tempo de aplicação da radioterapia e tempo de espera do paciente na fila).

\subsection{Discussão}

O Quadro 2 apresenta as perdas no fluxo do paciente quimioterápico e radioterápico e o Quadro 3 relaciona as técnicas enxutas implementadas no ICAVC, assim com os resultados obtidos com a aplicação destas. Cabe ressaltar que embora sejam possíveis dificuldades na implementação da PE quanto aos recursos materiais e à adaptação das técnicas enxutas, o hospital reconheceu como uma dificuldade, apenas a resistência dos funcionários às mudanças nos processos e à nova cultura organizacional.

As melhorias obtidas com a implementação das técnicas enxutas representaram ganhos para os fluxos dos pacientes quimioterápicos, radioterápicos e cirúrgicos, pois abrangeram vários processos comuns a estes.

A implementação da PE contou com participação e apoio da alta gestão em todo o processo, entretanto nos momentos iniciais ocorreu resistência dos 
funcionários, esta foi contornada por meio dos treinamentos e da conscientização dos potenciais benefícios da PE. O ICAVC buscou desenvolver a cultura de melhoria contínua.

Quadro 2 - Perdas no fluxo do paciente quimioterápico e radioterápico.

\begin{tabular}{|c|c|c|}
\hline Perdas & Descrição & Exemplos hospitalares \\
\hline Falhas & $\begin{array}{l}\text { Atividades que não são realizadas } \\
\text { corretamente. }\end{array}$ & $\begin{array}{c}\text { Extravio de prescrições médicas. } \\
\text { Não inclusão de quimioterapias no } \\
\text { faturamento. } \\
\text { Atraso no preparo dos } \\
\text { medicamentos. } \\
\text { Programação da quimioterapia não } \\
\text { considerava a capacidade do setor e } \\
\text { o tempo de infusão dos } \\
\text { medicamentos. } \\
\text { Ociosidade das poltronas da } \\
\text { quimioterapia. }\end{array}$ \\
\hline $\begin{array}{l}\text { Excesso de } \\
\text { processamento }\end{array}$ & $\begin{array}{l}\text { Produzir com o nível de qualidade } \\
\text { superior ao demandado ou realizar } \\
\text { uma atividade desnecessária. }\end{array}$ & $\begin{array}{l}\text { Funcionários procurando prescrições } \\
\text { médicas extraviadas. }\end{array}$ \\
\hline Espera & $\begin{array}{c}\text { Tempo no qual não é realizado nada } \\
\text { de produtivo. }\end{array}$ & $\begin{array}{l}\text { Pacientes esperando na fila pelo } \\
\text { procedimento quimioterapia e } \\
\text { radioterapia. } \\
\text { Pacientes esperando dentro da sala } \\
\text { de radioterapia. } \\
\text { Enfermeiras esperando no início do } \\
\text { turno da quimioterapia. }\end{array}$ \\
\hline
\end{tabular}

Fonte: Desenvolvido pela autora.

Quadro 3 - Técnicas enxutas utilizadas no hospital A no fluxo do paciente de quimioterapia (novos pacientes sem exames) e radioterapia

\begin{tabular}{|c|c|c|c|c|}
\hline $\begin{array}{c}\text { Processos do } \\
\text { hospital }\end{array}$ & Técnicas & Dificuldade & Resultados & $\begin{array}{l}\text { Número de } \\
\text { técnicas }\end{array}$ \\
\hline Quimioterapia & $\begin{array}{l}\text { Mapeamento do } \\
\text { fluxo de valor e } \\
\text { kaizen, } 5 \mathrm{~S}, \\
\text { nivelamento da } \\
\text { carga de } \\
\text { trabalho, } \\
\text { padronização, } \\
\text { trabalho em e } \\
\text { equipe e } \\
\text { trabalhadores } \\
\text { multifuncionais, } \\
\text { controle visual e } \\
\text { controle de } \\
\text { qualidade zero } \\
\text { defeitos }\end{array}$ & $\begin{array}{l}\text { Resistência dos } \\
\text { funcionários }\end{array}$ & $\begin{array}{l}\text { Aumento da } \\
\text { capacidade de } \\
\text { aplicação de } \\
\text { quimioterapia de } \\
\text { 23\%. Redução } \\
\text { do tempo de } \\
\text { espera do } \\
\text { paciente para } \\
\text { infusão da } \\
\text { quimioterapia } \\
\text { para 1h. } \\
\text { Redução do } \\
\text { tempo de } \\
\text { carregamento } \\
\text { das poltronas de } \\
40 \text { mim }\end{array}$ & 8 \\
\hline
\end{tabular}

Revista Produção Online. Florianópolis, SC, v. 18, n. 2, p. 593-619, 2018. 
Quadro 1 3- Técnicas enxutas utilizadas no hospital A no fluxo do paciente de quimioterapia (novos pacientes sem exames) e radioterapia

\begin{tabular}{|c|c|c|c|c|}
\hline $\begin{array}{l}\text { Processos do } \\
\text { hospital }\end{array}$ & Técnicas & Dificuldade & Resultados & $\begin{array}{l}\text { Número de } \\
\text { técnicas }\end{array}$ \\
\hline Triagem & $\begin{array}{l}\text { Mapeamento do } \\
\text { fluxo de valor, } \\
\text { kaizen, } \\
\text { nivelamento da } \\
\text { carga de } \\
\text { trabalho e } \\
\text { padronização } \\
\end{array}$ & $\begin{array}{l}\text { Resistência dos } \\
\text { funcionários }\end{array}$ & * & 4 \\
\hline APAC & $\begin{array}{l}\text { Mapeamento do } \\
\text { fluxo de valor, } \\
\text { kaizen, } \\
\text { padronização } \\
\end{array}$ & $\begin{array}{l}\text { Resistência dos } \\
\text { funcionários }\end{array}$ & $\begin{array}{c}\text { Redução de } \\
74 \% \text {, ou até } 28 \\
\text { dias no lead time } \\
\text { do paciente } \\
\end{array}$ & 3 \\
\hline Recepção & $\begin{array}{l}\text { Mapeamento do } \\
\text { fluxo de valor, } \\
\text { kaizen, } 5 \mathrm{~S}, \\
\text { padronização e } \\
\text { controle de } \\
\text { qualidade zero } \\
\text { defeitos. }\end{array}$ & $\begin{array}{l}\text { Resistência dos } \\
\text { funcionários }\end{array}$ & * & 6 \\
\hline $\begin{array}{l}\text { Controle de } \\
\text { prontuários }\end{array}$ & $\begin{array}{l}\text { Mapeamento do } \\
\text { fluxo de valor, } \\
\text { kaizen, } 5 \mathrm{~S}, \\
\text { padronização e } \\
\text { controle de } \\
\text { qualidade zero } \\
\end{array}$ & $\begin{array}{l}\text { Resistência dos } \\
\text { funcionários }\end{array}$ & * & 6 \\
\hline Laboratórios & $\begin{array}{l}\text { Mapeamento do } \\
\text { fluxo de valor, } \\
\text { kaizen, } 5 \mathrm{~S}, \\
\text { padronização e } \\
\text { controle de } \\
\text { qualidade zero } \\
\text { defeitos. } \\
\end{array}$ & $\begin{array}{l}\text { Resistência dos } \\
\text { funcionários }\end{array}$ & $\begin{array}{l}\text { Redução de } \\
90 \% \text { no prazo } \\
\text { dos exames. }\end{array}$ & 5 \\
\hline Farmácia & $\begin{array}{c}5 S, \\
\text { padronização e } \\
\text { controle de } \\
\text { qualidade zero } \\
\text { defeitos. }\end{array}$ & $\begin{array}{l}\text { Resistência dos } \\
\text { funcionários. }\end{array}$ & $\begin{array}{c}\text { Aumento da } \\
\text { produtividade na } \\
\text { manipulação dos } \\
\text { medicamentos. }\end{array}$ & 3 \\
\hline Radioterapia & $\begin{array}{c}\text { Mapeamento do } \\
\text { fluxo de valor, } \\
\text { kaizen, } \\
\text { padronização, } \\
\text { gestão visual, } \\
\text { 5S, TRF, } \\
\text { controle de } \\
\text { qualidade zero } \\
\text { defeitos } \\
\end{array}$ & $\begin{array}{l}\text { Resistência dos } \\
\text { funcionários. }\end{array}$ & $\begin{array}{l}\text { Redução do } \\
\text { tempo de espera } \\
\text { de } 50 \text { min para } \\
20 \text { min. } \\
\text { Criação de uma } \\
\text { recepção. }\end{array}$ & 7 \\
\hline
\end{tabular}

* Processos cujos resultados obtidos com a implementação das técnicas enxutas não foram detalhados.

Fonte: Desenvolvido pela autora

A sustentabilidade das melhorias foi obtida por meio da padronização das atividades, da definição de responsáveis, da definição de metas dos projetos, do acompanhamento dos indicadores de desempenho antes e após as intervenções de 
melhoria. Segundo a coordenadora de qualidade, a estrutura de implementação DMAIC proporciona a sustentabilidade das melhorias à medida que as melhorias projetadas são implantadas e posteriormente analisadas criticamente dando início a um novo ciclo de melhorias.

Assim como a literatura, o caso prático evidenciou que a implementação da PE no ICAVC abrangeu os fluxos de pacientes, materiais e informações. Nos processos de quimioterapia e radioterapia, o paciente é processado. Na farmácia e laboratórios ocorre o processamento de materiais. As informações são o foco da transformação nos processos de controle de prontuários, APAC, recepção e triagem.

Opondo-se as pesquisas de Souza (2009), Radnor, Holweg e Waring (2012), Burgess e Radnor (2013), o caso prático consistiu em implementação sistêmica e estruturada da PE, vinculada ao planejamento estratégico do hospital, alinhada com os objetivos operocionais e com o desenvolvimento da cultura de melhoria contínua.

Quanto a estrutura de implementação da PE, Bertani (2012) afirmou que muitas vezes são formadas equipes ad hoc e estabelecida uma equipe interna fixa para gerenciar todo o processo de implantação. Assim, no ICAVC foi verificado a formação de uma equipe fixa, (dois consultores da Hominiss e a coordenadora da qualidade) para conduzir os projetos de melhoria liderando a implantação da PE desde a fase inicial, assim como equipes ad hoc.

Concordando com Bertani (2012), no ICAVC o comprometimento dos funcionários foi o fator crítico na implementação da PE, o qual foi alcançado por meio dos treinamentos e apoio da alta gestão. A melhoria contínua ocorre em ciclos DMAIC, sendo as melhorias obtidas com a implantação das técnicas enxutas mantidas por meio da padronização

Contrapondo Bertani (2012), verificou-se que o ICAVC definiu objetivos e metas claras em cada projeto de melhoria em direção a mentalidade enxuta.

A semelhança das pesquisas de Teichgräber e Bucourt (2012), Dickson et al. (2009), Carter et al. (2012) e LaGanga (2011), no ICAVC as primeiras técnicas enxutas a serem implementadas foram o kaizen e MFV.

A partir do estudo de caso foi possível elaborar as seguintes diretrizes para implementação da PE em operações hospitalares.

1. A implementação da PE vinculada ao planejamento estratégico promove maior comprometimento dos profissionais envolvidos; 
2. A operação hospitalar pode contratar empresa de consultoria em Lean Healthcare.

3. A implementação das técnicas enxutas deve abranger fluxos de pacientes, informações e materiais, ou seja, processos de linha de frente ou retaguarda.

4. A método de melhoria contínua DMAIC pode ser utilizado na implementação das técnicas enxutas em um hospital;

5. Sugere-se iniciar a implantação da PE por um processo considerado problemático (ou gargalo), com alto volume ou crítico à rentabilidade do hospital;

6. A implantação da PE deve ser operacionalizada por meio de projetos de melhoria com escopo, participantes e metas definidas com o objetivo de garantir uma estrutura sistemática eficaz para a aplicação das técnicas enxutas;

7. Estruturar os treinamentos em duas partes, teórica e prática demonstrou ser eficaz na aplicação do conhecimento por meio dos projetos de melhoria;

8. Os projetos de melhoria devem ser iniciados com a implementação das técnicas kaizen e MFV;

9. As melhorias alcançadas com a implementação da PE podem ser mantidas por meio da padronização, dos indicadores de desempenho e de novos ciclos DMAIC;

10. Deve-se conciliar produtividade e qualidade. O foco em redução de custo é limitado pelas questões de qualidade dos serviços que afetam a segurança do paciente;

11. A implantação da PE deve contar com o forte apoio da gestão em todos os momentos;

12. O objetivo da implementação da PE deve ser a mudança cultural da operação hospitalar em direção a melhoria contínua dos processos.

\section{CONSIDERAÇÕES}

Este artigo analisou a implementação da PE em um hospital oncológico, proporcionando percepções para a identificação dos desperdícios no setor de saúde 
e para a eliminação ou redução destes por meio das técnicas enxutas.

A literatura sobre lean healthcare apresenta grande acervo de pesquisas no tema, entretanto estas pesquisas focam nas aplicações isoladas das técnicas enxutas e nos resultados obtidos. Assim, uma das contribuições teóricas deste artigo foi detalhar o caminho percorrido pelo hospital na implementação da PE, permitindo a compreensão da adaptação de algumas técnicas enxutas à processos hospitalares específicos. Baseando-se na literatura e no estudo empírico foi possível estabelecer doze diretrizes para implementação da PE sendo esta outra contribuição teórica do artigo.

A contribuição prática do artigo consiste em fornecer auxílio as operações hospitalares que desejarem implementar a cultura de melhoria enxuta, obtendo ganhos em qualidade e produtividade simultaneamente.

O desenvolvimento deste trabalho apresentou algumas limitações, sendo que uma delas consiste no fato de o estudo empírico ter sido realizado em uma única operação hospitalar e de ter abrangido apenas dois fluxos, quimioterapia e radioterapia.

Outra limitação foi a realização de entrevistas com apenas duas gestoras, uma vez que devido a rotina de trabalho e a indisponibilidade dos funcionários não foi possível realizar entrevistas em grupo por meio de grupos focais. Considerando as limitações apresentadas, a melhoria da pesquisa iniciada neste artigo e continuidade de pesquisas neste tema, recomenda-se o desenvolvimento das seguintes pesquisas:

- A replicação desta pesquisa em outras operações hospitalares, abrangendo outros fluxos de pacientes;

- Pesquisas focada em liderança e no papel dos recursos humanos envolvidos no processo de implementação da PE;

- O desenvolvimento de uma metodologia de referência para implementação da cultura enxuta;

- A implementação da PE na cadeia de suprimentos hospitalar. 


\section{REFERÊNCIAS}

ALLWAY, M.; CORBETT, S. Shifting to lean service: stealing a page from manufacturers playbooks. Journal of Organizational Excellence, v.21, n. 2, p. 45-54, 2002. DOI: $\underline{10.1002 / n p r .10019}$

ARAÚJO, C. A. S. Fatores a serem gerenciados para o alcance da qualidade para os clientes internos: um estudo em um conjunto de hospitais brasileiros. 2005. $414 \mathrm{f}$. Tese (Pós-Graduação de Administração) - Universidade Federal do Rio de Janeiro, Rio de Janeiro, 2005.

ARONSSON, H.; ABRAHAMSSON, M.; SPENS, K. Developing lean and agile health care supply chains. Supply Chain Management: an International Journal, v. 16, n. 3, p.176183, 2011. DOI: $\underline{10.1108 / 13598541111127164}$

BARLOW, G. L. Just-in-time: implementation within the hotel industry: a case study. International Journal of Production Economics, v. 80, n. 2, p. 155-167, 2002. DOI:doi.org/10.1016/S0925-5273(02)00315-8

BATAGLIA, F. Lean é sinônimo de facilidade em hospitais. Disponível em: <http://www.lean.org.br>. Acesso em: 12 abr. 2013.

BERTANI, T.M. Lean healthcare: recomendações para implantações dos conceitos de produção enxuta em ambientes hospitalares. 2012. 166f. Dissertação (Pós-graduação em Engenharia de Produção)- Escola de Engenharia de São Carlos da Universidade de São Paulo. São Carlos, 2012.

BURGESS, N.; RADNOR, Z. Evaluating lean in healthcare. International Journal of Health Care, Quality Assurance, v. 26, n. 3, p. 220-235, 2013.

BUSHELL, S.; SHELEST, B. Discovering lean thinking at progressive healthcare. The Journal for Quality and Participation, v. 25, n. 2, p. 20-5, 2002.

DOI: $10.1108 / 09526861311311418$

CARTER, P. M. et al. Optimizing clinical operations as part of a global emergency medicine initiative in Kumasi, Ghana: application of lean manufacturing principals to low-resource health systems. Academic Emergency Medicine, v. 19, n. 3, p. 338-347, 2012.

DOI: 10.1111/j.1553-2712.2012.01311.x

CAVALLINI, M. E.; BISSON, M. P. Farmácia hospitalar: Um enfoque em sistemas de saúde. 2. ed. Barueri: Manole, 2010.

CLARK, D. M.; SILVESTER, K.; KNOWLES, S. Lean management systems: creating a culture of continuous quality improvement. Journal of Clinical Pathology, v. 66, n. 8, p. 638-643, 2013. DOI: 10.1136/iclinpath-2013-201553

DICKSON, E. W. et al. Use of lean in the emergency department: a case series of 4 hospitals. Annals of Emergency Medicine, v. 54, n. 4, p. 504-510, 2009.

DOI: 10.1016/j.annemergmed.2009.03.024

FEINSTEIN, K. W.; GRUNDEN, N.; HARRISON, E. I. A region addresses patient safety. American Journal of Infection Control, v. 30, n. 4, p. 248-51, 2002.

DOI: http://dx.doi.org/10.1067/mic.2002.122645 
GRABAN, M. Hospitais Lean: melhorando a qualidade, a segurança dos pacientes e o envolvimento dos funcionários. Porto Alegre: Bookman, 2013

GODINHO FILHO, M. Paradigmas estratégicos de gestão da manufatura configuração, relações com o planejamento e controle da produção e estudo exploratório na indústria de calçados. 2004. 286 f. Tese (Doutorado em Engenharia de Produção) Universidade Federal de São Carlos, São Paulo, 2004.

HOPP. W. J.; LOVEJOY W. S. Hospital operations: principles of high efficiency health care. Upper Saddle River, NJ: Pearson FT Press, 2013.

JIMMERSON, C. Value stream mapping for healthcare made easy. New York: Productivity Press, 2010.

KOLLBERG, B.; DAHLGAARD, J. J.; BREHMER, P. O. Measuring lean initiatives in health care services: issues and findings. International Journal of Productivity and Performance Management, v. 56, n. 1, p.7-24, 2007. DOI:http://dx.doi.org/10.1108/17410400710717064

LA FORGIA, G. M.; COUTTOLENC, B. F. Desempenho hospitalar no brasil: em busca da Excelência. São Paulo: Singular, 2009.

LAGANGA, L. R. Lean service operations: reflections and new directions for capacity expansion in outpatient clinics. Journal of Operations Management, v. 29, n. 5, p. 422-433, 2011. DOI: $\underline{10.1016 / j . j o m .2010 .12 .005}$

LIKER, J. K. O modelo toyota: 14 princípios de gestão do maior fabricante do mundo. Porto Alegre: Bookman, 2008.

LIKER, J. K.; MORGAN, J. M. The Toyota way in services: the case of lean product development. Academy of Management Perspectives, v. 2, n. 20, p. 6-20, 2006. DOI: $\underline{\text { 10.5465/AMP.2006.20591002 }}$

LUONGO, J. Gestão de qualidade em saúde. São Paulo: Rideel, 2011.

MALAGÓN-LODOÑO, G.; MORERA, R. G.; LAVERDE, G. P. Administração hospitalar. 2.ed. São Paulo: Guanabara Koogan, 2000.

PAPADOPOULOS, T.; RADNOR, Z.; MERALI, Y. The role of actor associations in understanding the implementation of lean thinking in healthcare. International Journal of Operations \& Production Management, v. 31, n. 1-2, p. 167-191, 2011. DOI: $\underline{10.1108 / 01443571111104755}$

PHENG, L. S.; ARAIN, F. M.; FANG, J. W. Y. Applying just-in-time principles in the delivery and management of airport terminal buildings. Built Environment Project and Asset Management, v. 1, n. 1, p. 104-121, 2011. DOI:http://dx.doi.org/10.1108/20441241111143812

PIERCY, N.; RICH, N. Lean transformation in the pure service environment: the case of the call service centre. International Journal of Operations \& Production Management, v. 29, n. 1, p. 54-76, 2009. DOI: 10.1108/01443570910925361

RADNOR, Z. J.; HOLWEG, M.; WARING, J. Lean in healthcare: the unfilled promise? Social Science \& Medicine, v. 74, n. 3, p. 364-371, 2012. DOI:10.1016/j.socscimed.2011.02.011 
SHINGO, S. O Sistema Toyota de Produção do ponto de vista da engenharia de produção. Porto Alegre: Bookman, 1996.

SILVA, E. L.; MENEZES, E. M. Metodologia da pesquisa e elaboração de dissertação. Florianópolis: UFSC, 2005.

SOLIMAN, M. S.; SAURIN, T. A. Uma análise das barreiras e dificuldades em lean healthcare. Produção online, v. 17, n. 2, p. 620-640, 2017.

DOI: http://dx.doi.org/10.14488/1676-1901.v17i2.2605

SOUZA, L. B. Trends and approaches in lean healthcare. Leadership in Health Services, v. 22, n. 2, p. 121-139, 2009. DOI:http://dx.doi.org/10.1108/17511870910953788

SOUZA, V. H. S.; MOZACHI, N. O hospital: manual do ambiente hospitalar. 3. ed. Curitiba: Manual Real, 2009.

SULLIVAN, P. et al. Using lean methodology to improve productivity in a hospital oncology pharmacy. American Journal of Health-System, v.71, n.17, p. 1491-1498, 2014.

DOI: $\underline{10.2146 / a j h p 130436}$

TEICHGRÄBER, U. K.; BUCOURT, M. Applying value stream mapping techniques to eliminate non-value-added waste for the procurement of endovascular stents. European Journal of Radiology, v. 81, n. 1, p. 47-52, 2012. DOI: 10.1016/j.ejrad.2010.12.045

WARING, J. J.; BISHOP, S. Lean healthcare: rhetoric, ritual and resistance. Social Science \& Medicine, v. 71, n. 7, p. 1332-1340, 2010. DOI: 10.1016/j.socscimed.2010.06.028

WOMACK, J. P. et al. Going lean in health care. IHI Innovation Series white paper. Cambridge, MA: Institute for Healthcare Improvement, 2005. Disponível em:

<http://www.ihi.org>. Acesso em:23 abril 2013.

YASIN, M. M.; WAFA, M. A.; SMALL, M. H. Just-in-time implementation in the public sector. International Journal of Operations \& Production Management, v. 21, n. 9, p. 1195-1204, 2001.

DOI:http://dx.doi.org.ez15.periodicos.capes.gov.br/10.1108/EUM0000000005925

YEH, H. et al. Applying lean six sigma to improve healthcare: an empirical study. African Journal of Business Management, v. 5, n. 31, p. 12356-12370, 2011. DOI:http://dx.doi.org.ez15.periodicos.capes.gov.br/10.1108/EUM0000000005925

YIN, R. Estudo de caso: planejamento e método. Porto Alegre: Bookman, 2005.

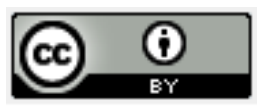

Artigo recebido em 14/05/2017 e aceito para publicação em 10/10/2017 DOI: http://dx.doi.org/10.14488/1676-1901.v18i2.2873 